\title{
Büchter, Karin
}

\section{Zum Gehalt berufs- und wirtschaftspädagogischer Selbstthematisierungen}

\section{- Rückblick und Ausblick}

Seifried, Jürgen [Hrsg.]; Seeber, Susan [Hrsg.]; Ziegler, Birgit [Hrsg.]: Jahrbuch der berufs- und wirtschaftspädagogischen Forschung 2017. Opladen ; Berlin ; Toronto : Verlag Barbara Budrich 2017, S. 11-28. (Schriftenreihe der Sektion Berufs- und Wirtschaftspädagogik der Deutschen Gesellschaft für Erziehungswissenschaft (DGfE))

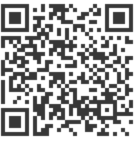

Quellenangabe/ Reference:

Büchter, Karin: Zum Gehalt berufs- und wirtschaftspädagogischer Selbstthematisierungen -

Rückblick und Ausblick - In: Seifried, Jürgen [Hrsg.]; Seeber, Susan [Hrsg.]; Ziegler, Birgit [Hrsg.]: Jahrbuch der berufs- und wirtschaftspädagogischen Forschung 2017. Opladen ; Berlin ; Toronto : Verlag Barbara Budrich 2017, S. 11-28 - URN: urn:nbn:de:0111-pedocs-184146 - DOI: 10.25656/01:18414

https://nbn-resolving.org/urn:nbn:de:0111-pedocs-184146

https://doi.org/10.25656/01:18414

in Kooperation mit / in cooperation with:

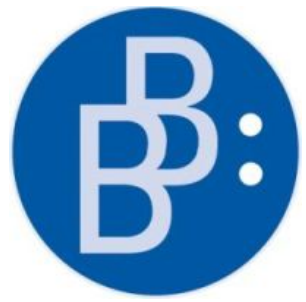

https://www.budrich.de

\section{Nutzungsbedingungen}

Gewährt wird ein nicht exklusives, nicht übertragbares, persönliches und beschränktes Recht auf Nutzung dieses Dokuments. Dieses Dokument ist ausschließlich für den persönlichen, nicht-kommerziellen Gebrauch bestimmt. Die Nutzung stellt keine Übertragung des Eigentumsrechts an diesem Dokument dar und gilt vorbehaltlich der folgenden Einschränkungen: Auf sämtlichen Kopien dieses Dokuments müssen alle Urheberrechtshinweise und sonstigen Hinweise auf gesetzlichen Schutz beibehalten werden. Sie dürfen dieses Dokument nicht in irgendeiner Weise abändern, noch dürfen Sie dieses Dokument für öffentliche oder kommerzielle Zwecke vervielfältigen, öffentlich ausstellen, aufführen, vertreiben oder anderweitig nutzen

Mit der Verwendung dieses Dokuments erkennen Sie die Nutzungsbedingungen an.

\section{Terms of use}

We grant a non-exclusive, non-transferable, individual and limited right to using this document.

This document is solely intended for your personal, non-commercial use. Use of this document does not include any transfer of property rights and it is conditional to the following limitations: All of the copies of this documents must retain all copyright information and other information regarding legal protection. You are not allowed to alter this document in any way, to copy it for public or commercial purposes, to exhibit the document in public, to perform, distribute or otherwise use the document in public.

By using this particular document, you accept the above-stated conditions of use.

\section{Kontakt / Contact:}

\section{peDOCS}

DIPF | Leibniz-Institut für Bildungsforschung und Bildungsinformation Informationszentrum (IZ) Bildung

E-Mail: pedocs@dipf.de

Internet: www.pedocs.de

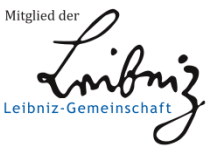


Schriftenreihe der Sektion

Berufs- und Wirtschaftspädagogik

der Deutschen Gesellschaft

für Erziehungswissenschaft (DGfE) 
Jürgen Seifried

Susan Seeber

Birgit Ziegler (Hrsg.)

\section{Jahrbuch der berufs- und} wirtschaftspädagogischen Forschung 2017

Verlag Barbara Budrich

Opladen • Berlin • Toronto 2017 
Bibliografische Information der Deutschen Nationalbibliothek

Die Deutsche Nationalbibliothek verzeichnet diese Publikation in der Deutschen

Nationalbibliografie; detaillierte bibliografische Daten sind im Internet über

http://dnb.d-nb.de abrufbar.

Gedruckt auf säurefreiem und alterungsbeständigem Papier

Alle Rechte vorbehalten

(C2017 Verlag Barbara Budrich, Opladen, Berlin \&Toronto

www.budrich-verlag.de

ISBN 978-3-8474-2141-2 (Paperback)

eISBN 978-3-8474-1131-4 ( eBook)

Das Werk einschließlich aller seiner Teile ist urheberrechtlich geschützt. Jede Verwertung außerhalb der engen Grenzen des Urheberrechtsgesetzes ist ohne Zustimmung des Verlages unzulässig und strafbar. Das gilt insbesondere für Vervielfältigungen, Übersetzungen, Mikroverfilmungen und die Einspeicherung und Verarbeitung in elektronischen Systemen.

Umschlaggestaltung: Bettina Lehfeldt, Kleinmachnow - www.lehfeldtgraphic.de Typographisches Lektorat: Anja Borkam, Jena 


\section{Inhaltsverzeichnis}

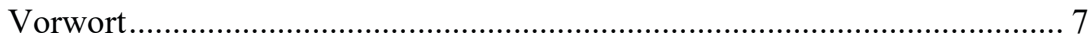

\section{Teil I: Überlegungen zum disziplinären Selbstverständis der Berufs- und Wirtschaftspädagogik}

Karin Büchter

Zum Gehalt berufs- und wirtschaftspädagogischer

Selbstthematisierungen - Rückblick und Ausblick

Matthias Söll

Orientierungspotenziale des Basiscurriculums der Berufs- und

Wirtschaftspädagogik für die disziplinäre Binnen- und Außenlegitimität.... 29

\section{Teil II: Berufliche Lehr-Lern- und Unterrichtsforschung}

Christoph Helm, Jacqueline Netzthaler und Bettina Kreuzer

Kooperatives Lernen im kaufmännischen Unterricht. Eine

Netzwerkanalyse zu sozial-konstruktivistischen Lerntheorien 43

Manuela Niethammer und Anke Langner

Inklusion als fachdidaktischer Anspruch

Svenja Ohlemann und Katja Driesel-Lange

Individuelle Begleitung beruflicher Entwicklung: Kompetenzförderung anhand von Lernstilen.

Mandy Hommel, Bärbel Fürstenau, Claudia Leopold, Héctor Ponce und Mario López

Beitrag von Banken-Webseiten zur Entwicklung der Finanzkompetenz

potentieller Darlehensnehmer/innen über Baufinanzierungen

\section{Teil III: Hochschul- und Lehrerbildungsforschung}

Silke Lange und Dietmar Frommberger

Zur Ausgestaltung schulischer Praxisphasen im beruflichen

Lehramtsstudium - Ergebnisse einer ersten Analyse. 
Heike Jahncke und Karina Kiepe

Handlungsempfehlungen aus dem Einsatz und der Evaluation eines

Tagungsportfolios im Rahmen der Lehrerbildung

Julia Warwas und Andreas Rausch

Unterrichtliche Überzeugungen und Praktiken von Lehrkräften an

Beruflichen Oberschulen - eine fächervergleichende Analyse....

Christian Schmidt

Die Öffnung des Hochschulzugangs für beruflich Gebildete: Förderung studienrelevanter Schlüsselkompetenzen in der Studieneingangsphase am Beispiel der Universität Kassel

\section{Teil IV: Schulentwicklungsforschung}

Marc Casper, Bernadette Dilger, Frederik Fischer, Katharina Fütterer, Nicole Naeve-Stoß und Tade Tramm

Entwicklung beruflicher Schulen im regionalen Verbund.....

Herausgeberschaft.

Autorinnen und Autoren 


\title{
Zum Gehalt berufs- und wirtschaftspädagogischer Selbstthematisierungen - Rückblick und Ausblick
}

\author{
Karin Büchter
}

\section{Einleitung}

Während in Einführungsschriften, Handbüchern, Festschriften und Aufsätzen kontinuierlich Aussagen zum berufs- und wirtschaftspädagogischen Selbstverständnis getroffen werden, finden Kell $(2014,53)$ zufolge, direkte Diskurse und gemeinsame Bilanzierungen über das Selbstverständnis nicht statt. Das Ausbleiben von solchen Diskursen kann ein Zeichen für einen fehlenden disziplinären Problemdruck sein oder aber für bereits verhärtete Abschottungen von Positionen und Forschungsrichtungen, die für elementare Inhalte und Fragen von Selbsthematisierungen blind gemacht haben. Im folgenden Beitrag geht es um die Frage, welchen Verlauf die berufs- und wirtschaftspädagogische Selbstverständnisfrage genommen hat, welche neuralgischen Punkte und kategorialen Bezüge dabei zur Diskussion standen und stehen sollten.

\section{Aufbruchsstimmungen, Kontroversen und Krisen}

Die Selbstverständnisfrage beginnt am Anfang des 20. Jahrhunderts mit der disziplinären Sichtbarwerdung im Zuge der Errichtung von Lehrstühlen, der Publikation von Grundlagentexten im sich entfaltenden Fachschrifttum und von Einführungsschriften, der Suche nach einer disziplinären Bezeichnung, der Festlegung von Grundkategorien und inhaltlichen Schwerpunkten. Wesentliche Kriterien der Selbstverständnisfrage waren von Anfang an die disziplinäre Eigenständigkeit und Einheitlichkeit. In seiner „Berufsschul- und Wirtschaftspädagogik“ ging es Feld $(1928,54)$ darum, einen ,selbständigen pädagogischen Wissenschaftszweig" aufzubauen. Dieser sollte sich von den anderen ,grundlegend durch Stoffabgrenzung, Methode und Erkenntnisziel“ (ebd.) und durch ,seine besonderen Fragestellungen, die nicht mit den Mitteln bisheriger pädagogischer Einstellung beantwortet werden können“ (ebd.), unterscheiden. Ihm war außerdem daran gelegen, dass sich die „Berufsschul- und Wirtschaftspädagogik“ von einer schulbezogenen Anwendungsdisziplin zu einer wissenschaftlichen Disziplin entwickelt, die im Theoriebereich eine „selbstständige Geltung im Rahmen der Gesamtpädagogik“ 
(V) erlangt. Die damaligen Überlegungen waren an der Kulturphilosophie und Berufsbildungstheorie orientiert, jedoch mit überwiegend axiomatischdeduktiver und dezisionistischer Ausrichtung. Die Verkürzung hermeneutischer Interpretationen von Mensch, Kultur und Gemeinschaft auf programmatische Begriffe wie „Volk“ und „Nation“ begünstigten schließlich den fließenden Übergang der ersten theoretischen Ansätze dieser noch identitätssuchenden Disziplin in die nationalsozialistische Ideologie. ${ }^{1}$ So fand die „endgültige Durchsetzung der Wirtschaftspädagogik als selbständig vertretene Hochschuldisziplin“ (Pleiß 1973, 209 ff.) unter der NS-Diktatur statt. Eine kritische Reflexion ideologischer Funktionalisierbarkeit der Disziplin erfolgte erst viel später und auch nur im kleinen Umfang. ${ }^{2}$ Wie in anderen erziehungswissenschaftlichen Disziplinen wollte man sich nach 1945 durch eine an der Realität orientierte, möglichst objektive wissenschaftliche Ausrichtung der Berufs- und Wirtschaftspädagogik von dem Vorwurf politischideologischer Verführbarkeit befreien. So sprach Schlieper $(1949,42)$ von einer ,bedeutsame[n] Wandlung in der Auffassung der wirtschaftspädagogischen Wissenschaft" und zwar von einer ,primär normativen zu einer vorwiegend empirischen“ (ebd.). Der Wissenschaftscharakter sollte durch eine stärker induktive und empirisch-phänomenologische Vorgehensweise bestimmt sein, die sich dem ,ganzen Menschen entsprechend seiner Bestimmungen in einer [...] bestimmten Lebenssituation" (Schlieper 1954/1967, 72) verpflichtet fühlt, und danach fragt, "welche erzieherischen Probleme“ dadurch entstehen, daß der ,in der Wirtschaft wirkende Mensch [...] einen erheblichen Teil seines Lebens in der Wirtschaft zubringt" (Abraham 1953, 22). Obwohl im Laufe der 1950er Jahre unterschiedliche empirische Untersuchungen (vgl. Dörschel 1960, 23) durchgeführt wurden, entfachte sich am Ende dieses Jahrzehnts eine ,wissenschaftliche Kritik an der Wirtschaftspädagogik“ (Linke 1961, 737), und zwar ,sowohl im internen Kreis der Fachvertreter als auch in der fachfremden Öffentlichkeit“ (ebd.), die „mit zum Teil erheblichem emotionalen Engagement" (Kiehn 1962/67, 36) geführt wurde. Fragen nach dem wissenschaftlichen und disziplinpolitischen Selbstverständnis beschäftigten aber die Erziehungswissenschaft insgesamt. Und ähnlich wie hier bildeten sich auch in der Berufs- und Wirtschaftspädagogik bis in die 1970er Jahre hinein unterschiedliche wissenschaftstheoretische und methodologische Positionen heraus (vgl. Dörschel 1960; Abel 1963; Zabeck 1972; Lipsmeier 1972; Lange 1972), über deren Grundannahmen und praktischen Implikationen immer auch im Zusammenhang mit der Selbstverständnisfrage gestritten wurde. Diese Kontroversen ließen aber keinen Zweifel an der Existenzberechtigung der Disziplin, da sich die Berufs- und Wirt-

1 „Ohne Zweifel vollzog sich damit eine Abwendung von der kulturphilosophisch-humanistischen Berufsbildungstheorie; an die Stelle der kulturpädagogischen trat eine politisch fundierte Erziehungstheorie“" (Pleiß 1971b, 206).

2 Siehe Neumann 1969; Pleiß 1973; Seubert 1977; Kipp \& Miller-Kipp 1994. 
schaftspädagogik wissenschaftsgeschichtlich bis dahin behauptet hatte (vgl. Pleiß 1973). Trotzdem gelang es in den Folgejahren nicht, den Diskurs der 1960er/1970er Jahre zu kultivieren und zu etablieren, stattdessen provozierte die weitere disziplinäre Binnendifferenzierung eine erneute Krisenstimmung, die man, so Stratmann (1979/1999, 509), durchaus als „Unsicherheit im Selbstverständnis der Berufs- und Wirtschaftspädagogik“ deuten könnte. Die verschiedenen Forschungsrichtungen könnten aber auch Ausdruck wissenschaftlichen „Reichtums“, der „Offenheit“ und „Lebendigkeit dieser Disziplin“ (ebd.) sein. Darum seien die erkannten „Identitätsprobleme alles andere als ein Krisensymptom" (ebd.). Aber ähnlich wie in der Erziehungswissenschaft wurde auch in Folgejahren die innere disziplinäre Vielfalt nicht als konstruktiv zu bearbeitendes Phänomen, sondern eher als „Systemproblem“ (Tenorth 2004, 382) begriffen. Ohne im Einzelnen wissenschaftshistorisch und -theoretisch zu erläutern, was disziplinäre Einheit ausmacht und welches ihre Vorteile sind, kritisierte die Deutsche Forschungsgemeinschaft (1990, $18 \mathrm{f}$.), dass ,die Berufs- und Wirtschaftspädagogik [...] bislang noch nicht zu einem einheitlichen Wissenschaftsverständnis gefunden [hat]". Da eine ausführliche Verständigung über Einheit und Idendität zu dieser Zeit fehlte, mussten disziplinexterne arbeitsmarkt- und berufsbildungspolitische Entwicklungen der 1990er Jahre, die als „Krise des Berufs“ und „Krise der Lehrerbildung" thematisiert wurden, zwangsläufig in die Frage münden: „Wird die Berufs- und Wirtschaftspädagogik auch zukünftig benötigt werden?" (Schmiel \& Sommer 2001, 18). Parallel zu solchen disziplinären Unsicherheiten, die seit dem Ende der wissenschaftstheoretischen und methodologischen Kontroversen der 1970er Jahre eher deprimiert und hilflos vorgetragen wurden, hat sich die Disziplin intern immer weiterentwickelt. Obwohl genaue szientometrische Analysen über die strukturelle und inhaltliche Differenzierung der Berufs- und Wirtschaftspädagogik noch ausstehen, ist deutlich, dass sich theoretische Positionen und Bezüge, Forschungsinteressen, methoden und -instrumente ausgeweitet haben. Gegenstände werden nicht mehr nur aus der Praxis gewonnen, sondern auch konstruiert, und zwischen Alltagserfahrungen in Arbeit, Beruf und Berufsbildung und wissenschaftlichem berufs- und wirtschaftspädagogischen Wissen sind immer mehr Betrachtungsebenen entstanden. Ein Diskurs quer zu den Paradigmen blieb aber aus, eher verstärkte sich eine Abschottung von Theorien und Forschungsschwerpunkten, wodurch auch das Interesse an Auseinandersetzungen mit der „Krisenanfälligkeit“ (Lisop 2009), den „,chronischen Krankheiten“ (Lempert 2009) oder der Tatsache, dass die Berufs- und Wirtschaftspädagogik „immer noch keinen disziplinären Ort gefunden“ (Bank 2009) habe, gering blieb. 


\section{Neuralgische Punkte in der Selbstverständnisfrage}

Um das Selbstverständnis diskutierbar zu machen, werden im Folgenden auf der Basis bisheriger Selbstthematisierungen neuralgische Punkt der Disziplin rückblickend und ausblickend betrachtet.

\subsection{Appendix der Erziehungswissenschaft?}

Das Verhältnis zwischen Berufs- und Wirtschaftspädagogik und Erziehungswissenschaft war nie eindeutig. Schon in ihrer Konstitutionsphase stand die damalige Berufs- und Wirtschaftspädagogik mit ihrer auf Beruf und Wirtschaft bezogenen Vorstellung von Pädagogik gegen die neuhumanistische Idee der zweckfreien Bildung. Die ersten Lehrstühle wurden auch nicht an philosophischen oder pädagogischen Fakultäten errichtet (vgl. Stratmann \& Bartel 1975, XV). Die Berufs- und Wirtschaftspädagogik kam erst über den Weg der Fachwissenschaften und der fachdidaktischen Notwendigkeit einer Ausbildung von Berufsschullehrern zur Pädagogik. Die Wirtschaftspädagogik hat sich ab 1923 mit eigenen Lehrstühlen zunächst als Handelsschulpädagogik aus den Handelswissenschaften der Handelshochschulen entwickelt. Um 1930 wurde sie zu einer eigenständigen Disziplin und schöpfte ihren Erkenntnisgegenstand nicht ausschließlich und vorrangig aus der erziehungswissenschaftlichen Theorie, sondern auch aus der Wirtschafts- und Sozialpolitik (vgl. Schürholz 1928), der Psychologie (vgl. Kautz 1929) und der Betriebswirtschaftslehre (vgl. Krasensky 1935). Die Gewerbelehrerbildung fand seit Ende des 19. Jahrhunderts zunächst in Gewerbelehrer-Seminaren, später an Berufspädagogischen Instituten, seit den 1920er Jahren auch an Technischen Hochschulen (Karlsruhe 1922, Dresden 1923, Stuttgart 1924, Braunschweig 1925) und Universitäten (Jena 1924, Hamburg 1930) statt, „wobei an den Universitäten die Pädagogik für Gewerbelehrer stärkeres Gewicht erhielt als an den Technischen Hochschulen" (Baumgardt 1976, 11). Nach Abel wurde das Grundwort Pädagogik dem Bestimmungswort Beruf erstmals 1921 im Rahmen einer „Berufspädagogischen Woche“ in Berlin zugeordnet (vgl. Abel 1963, 9). Die Übernahme der Begriffe Pädagogik und zunehmend auch Erziehungswissenschaft durch die gesamte Lehrerbildungsbewegung zu Beginn des 20. Jahrhunderts veranlasste auch die damalige Berufs- und Wirtschaftspädagogik dazu, sich stärker erziehungswissenschaftlich zu orientieren. Hierbei ist zu berücksichtigen, dass in dieser Zeit auch die Erziehungswissenschaft um ihre Autonomie rang und im Wissenschaftssystem selber noch umstritten war (vgl. Tenorth 2004, 359 f.). Dennoch war ihr Einfluss auf die berufs- und wirtschaftspädagogischen Denkformen deutlich. Für Feld (1928) stand fest, dass ,die Berufsschul- und Wirtschaftspädagogik“ eine „auf kulturphilosophischer Basis ruhende erziehungswissenschaftliche 
Disziplin“ (S. 53) ist. Ein Grundsatzstreit darüber, ob die Berufs- und Wirtschaftspädagogik überhaupt als Erziehungswissenschaft möglich sei, entfachte sich in den 1960er Jahren. Hatte Schlieper $(1952,423)$ die Wirtschaftspädagogik noch als „Wirtschafts- und Sozialwissenschaft mit erziehungswissenschaftlicher Schau“ bezeichnet, wies Linke $(1961,737)$ auf die Tendenz der Wirtschaftspädagogik ,zu einem mechanistischen und instrumentalistischen Erziehungsdenken“ hin und warnte davor, dass sie sich ,in ihren Formulierungen und Vorstellungen vom bisher üblichen Erziehungsdenken entfernt“ (ebd.). Deshalb sah er es als Aufgabe an, ,die Absonderung der Wirtschaftspädagogik von der Pädagogik überwinden zu helfen“ (ebd., 738). Die Befürchtungen gingen also dahin, die Disziplin könnte den Anschluss an die Erziehungswissenschaft verlieren. „Es ist kaum zuviel gesagt, daß die Wirtschaftspädagogik auf dem Wege zum Selbstverständnis [...] ziemlich weit hinter den Klärungen der allgemeinen Pädagogik hinterherhinkt" (Schwarzlose 1961, 766). Zu dieser Zeit hatte sich die Berufs- und Wirtschaftspädagogik noch nicht als Doppeldisziplin konsolidiert, so dass es auch um die Frage ging, ob eher die Wirtschafts- oder die Berufspädagogik als Pädagogik zu bezeichnen sei. Groothoff (1961) wies darauf hin, dass sich die Wirtschaftspädagogik ,anders als alle anderen besonderen Pädagogiken, anders auch [...] als die ,Berufs'-Pädagogik [...] als Sezession konstituiert" hat (S. 780). Deutlich würde, dass sich auch ,die Allgemeine Pädagogik, und zwar seit langem, fast ausschließlich mit der Berufspädagogik [...] auseinander[setzt]“" (S. 774). Der Wirtschaftspädagogik fehle die Historisierung der wirtschaftlichen, gesellschaftlichen und technologischen Phänomene, eine „Situationsanalyse“ in Form von Gesellschafts- und Kulturkritik sowie eine „humane Bestimmung der Wirtschaft" (S. 781). Auch Ritzel $(1961,753)$ war davon überzeugt, „daß es nur eine legitime Wirtschaftspädagogik gibt, eben die Berufspädagogik“: „Wir bestreiten den Erkenntnisanspruch und Wissenschaftscharakter der Wirtschaftspädagogik zugunsten dessen der Berufspädagogik [...]. Sie [die Berufspädagogik] stellt die Fragen, zu denen sich die Frage der einen und ganzen Pädagogik im Hinblick darauf differenziert, daß der, Weg zu der höheren Allgemeinbildung über den Beruf und nur über den Beruf" führt" (ebd.). Der zweite umfassendere Selbstverständnisdiskurs problematisierte erneut das Verhältnis zwischen Erziehungswissenschaft und Berufs- und Wirtschaftspädagogik. Während Lange (1971/1975, 308) feststellte, dass „kein Zweifel darüber bestehen [kann], daß der Forschungsstand [...] noch größer ist als sonst in der deutschen Erziehungswissenschaft", kritisierte Lipsmeier $(1972,22)$, dass ,die Rezeption des wissenschaftstheoretischen und erziehungswissenschaftlichen Standards [...] so gut wie ausgeblieben [sei]“. Zabeck (1972) resümierte, die Berufs- und Wirtschaftspädagogik habe „keine eigene Methode, keine eigene Terminologie, keinen eigenen Theoriebegriff" (S. 586) und müsse daher auf die erziehungswissenschaftliche Grundlagendiskussion zurückgreifen (S. 585), jedoch dürfe nicht 
verkannt werden, dass es die Erziehungswissenschaft selber nicht vermocht hat, „einen Konsens über ihren Wissenschaftscharakter herbeizuführen“ (S. 577) und „,von einer Konsolidierung ihres Selbstverständnisses [...] weit entfernt" (ebd.) ist. Am Ende dieser Auseinandersetzung stand fest, dass die Berufs- und Wirtschaftspädagogik nicht in der Lage ist, von sich aus und innerhalb ihres eigenen Radius Gesellschafts- und Bildungsfragen zu beantworten. Ohne erziehungswissenschaftliche Anbindung laufe sie Gefahr, zur didaktischen Variante der Fachwissenschaften zu degradieren und die angehende Lehrerschaft ohne gesellschaftskritische Reflexion auf ihre Tätigkeit vorzubereiten (vgl. Stratmann \& Bartel 1975, XIII f.). Inwieweit es der damaligen Berufs- und Wirtschaftspädagogik tatsächlich gelungen ist, deutlich zu machen, dass die Erziehungswissenschaft auch auf sie angewiesen ist, ist bis heute nicht klar. Doch nach wie vor klammert die Erziehungswissenschaft die berufliche Integration der Jugend in die Arbeitswelt, die pädagogische Bedeutung von Beruf, Arbeit, Wirtschaft und Technik und die Funktionsweisen von Berufsbildung aus ihren Reflexionen und Untersuchungen weitgehend aus (vgl. ebd.).

Mit dem Vorsatz einer intensiven Verständigung über erziehungswissenschaftliche und berufs- und wirtschaftspädagogische Ziele, Vorgehensweisen und Praktiken wurde die Berufs- und Wirtschaftspädagogik auf der DGfETagung am 10. April 1972 in Nürnberg der Erziehungswissenschaft formal zugeordnet. Auch wenn es immer mal wieder Überlegungen dazu gab, wie das Verhältnis zwischen Erziehungswissenschaft und Berufs- und Wirtschaftspädagogik gestaltet werden kann, sind bis heute das Verhältnis und seine historischen Hypotheken, die Anlässe und Schwerpunkte diskursiver Wechselbeziehungen, gemeinsame Grundverständnisse, ebenso wie gegenseitige Zuschreibungen und Abgrenzungen noch nicht ausführlich aufgearbeitet worden.

\subsection{Objektivitätsanspruch und Normativitätsdilemma}

Ein historisch kontinuierliches Bemühen der Berufs- und Wirtschaftspädagogik besteht darin, in ihrer wissenschaftlichen Ausrichtung nicht als zu normativ und anwendungsorientiert dazustehen, sondern paradigmatisch offen $\mathrm{zu}$ sein. So heißt es bei Feld $(1928,54)$ : „Was den wissenschaftlichen Aufbau betrifft, können wir hier [...] drei Wege gehen, spekulativ, empirisch und beides vereint. Für unsere beruflich eingestellte Pädagogik haben wir uns ohne weiteres für das letzte entschieden". Gedacht war also an eine Verbindung von empirischen Erkenntnissen mit pädagogischen Werten. Eine solche Kombination hatte auch Dörschel $(1960,30)$ vor Augen. Aufgrund ihres „empirisch-deskriptiv-explikativen“ Verfahrens sei „die Wirtschaftspädagogik [...] auf dem Weg zu einer eigenständigen Tatsachenforschung, ohne die 
normative, die begriffsorientierte erziehungsphilosophische, die vergleichende und historische Forschung zu vernachlässigen". Da sich das Normative aber nicht mit dem Anspruch reiner Wissenschaftlichkeit vertrug, die Berufsund Wirtschaftspädagogik sich andererseits auch als pädagogischpraxisbezogene Disziplin verstehen musste, stellte sich die Verknüpfung als nicht widerspruchsfrei dar. Für Zabeck $(1968,92)$ sollte sich die Wissenschaftlichkeit ,allein in der Hingabe, mit der der einzelne Fachvertreter der Idee der Wahrheit dient“, „,im Streben nach ,intellektueller Redlichkeit“ und ,Wahrhaftigkeit““, im Widerstreben „der ,pateiischen Argumentation““ und in der Mitteilung der Erkenntnisse ,in ,rationaler Zucht und begrifflicher Sauberkeit'“ äußern. Eine Normgebundenheit holte ihn aber dann mit seiner axiomatisierten Berufs- und Wirtschaftspädagogik wieder ein (ebd., 594 ff.). Die gesellschaftskritische und emanzipatorische Berufspädagogik richtete das Erkenntnisinteresse ihrer Forschung gezielt auf Herrschaftskritik, die Förderung von Selbstbestimmung und die Beseitigung von Minderprivilegierung (vgl. Lempert 1972). Vor dem Hintergrund des Werturteilstreits in den Sozialwissenschaften kam es schließlich auch in der Berufs- und Wirtschaftspädagogik zu unversöhnlichen Auffassungen von Wissenschaftlichkeit und Erkenntnisinteressen. Seit den 1990er Jahren war es dann die Modellversuchsforschung, die Kontroversen um das berufs- und wirtschaftspädagogische Methodologieverständnis zwischen angewandter und Grundlagenforschung provoziert hat (vgl. Beck 2003). Mit der überwiegend quantitativ ausgerichteten „empirischen Bildungsforschung“ seit Beginn der 2000er Jahre gab es für die Berufs- und Wirtschaftspädagogik einen Anlass, sich dem Verdacht einer überwiegend anwendungsorientieren Disziplin zu entledigen. Neben der bereits gut situierten empirischen Lehr- Lernforschung konnte nun die empirische Kompetenzforschung eine weitere Alternative zur Anwendungsforschung sein, da es hier um objektive Empirie gehen sollte. Doch was der Modellversuchsforschung an Theorie-, Empiriedefiziten und vereinfachter Modelltechnokratie vorgeworfen wurde, gilt ähnlich für die Kompetenzforschung. Wie in der empirischen Bildungsforschung war auch in der Berufs- und Wirtschaftspädagogik mit der Kompetenzorientierung von vornherein eine ,symbiotische[n] Beziehung des Kompetenzbegriffs zu diagnostischen Messverfahren“ (Dammer 2015, 125) gegeben. Die für theoretische Grundlegung herangezogenen erziehungswissenschaftlichen Kompetenztheorien wie die von Heinrich Roth werden pragmatisch so verkürzt, dass sie den methodischen Messmöglichkeiten und Messzielen angepasst werden können. Bildungsphilosophische und -theoretische Einwände gegen gesellschaftsunkritische und subjekttheoriearme Kompetenzverständnisse, gegen die weitgehende Reduktion von Kompetenzen auf Können, gegen die sozialschichtspezifische Gebundenheit von Kompetenzanforderungen und gegen die Auffassung von Lernsubjekten „als kausal funktionierende Apparaturen“ (ebd.) haben bislang kaum Eingang in die berufs- und wirtschaftspädagogische 
Kompetenzforschung gefunden; ${ }^{3}$ auch nicht die Frage, ob und inwieweit eine reflexive pädagogische Professionalisierung herkömmlicher und schulalltäglichen Kompetenzdiagnostik im Rahmen von Lehrertätigkeit nicht sinnvoller mit der Lehrerbildung verknüpft werden könnte als schulexterne Kompetenzmessungen, deren praktische und didaktische Wirksamkeit ohnehin fraglich ist. Schließlich steht auch die empirische Kompetenzforschung, die sich als wertfrei verstehen möchte, vor Objektivitätsgrenzen und einem Normativitätsdilemma, da sie die Bezugsgrößen ihrer Messung (Anforderungen der Arbeitswelt, curriculare Vorgaben) unhinterfragt setzt. Übersehen wird dabei das, was Heid $(1977,836)$ zu Bedenken gab, „daß es zwischen den genannten Gliedern des ,Ableitungszusammenhangs' logisch, theoretisch und empirisch begründ- und überprüfbare Vermittlungsprozesse und -entscheidungen höchster Komplexität gibt“, die zu den Fragen führen: „Welches Merkmal [...] wird warum als ,Anforderung' selegiert und definiert; warum bleiben andere Merkmale außer Betracht? Welche Anforderung wird warum als erhebungs- bzw. erziehungsrelevant ausgewählt? ([...] Welches Verhalten wird warum jeweils als anforderungsbedeutsam postuliert und welches Verhalten dann tatsächlich jeweils als Erfüllung (oder Verfehlung) der Anforderung honoriert (oder sanktioniert)?" (ebd.). Die empirische Kompetenzforschung ist hier begründungspflichtig, wenn sie sich nicht den Vorwurf gefallen lassen will, ihr Erhebungsinstrumentarium aus fraglosen Plausibilitäten abgeleitet zu haben, einem Mangel an kritischer Distanz zur eigenen Praxis und einer Verflochtenheit der Forschung mit politischen Forschungsfördertrends und deren Sachgesetzlichkeiten, die eine Ökonomie des Sehens erforderlich machen, zu unterliegen.

\section{3 „Politische Askese“, politische Steuerung und Diskursregime}

Trotz des Vorsatzes „,politischer Askese“ (Zabeck 1972, 581) steht Wissenschaft immer in einem gesellschaftlich-politischen Kontext (vgl. Albert 1991, 36). Sie setzt einen Sinnzusammenhang voraus, der gesellschaftlich bestimmt ist und damit in Entstehung und Vollzug abhängig von Politik. Gleichzeitig liefert sie der Politik mit ihren wissenschaftlichen Ergebnissen Argumente und Legitimationen. So gerät auch , die Berufs- und Wirtschaftspädagogik zur Sicherung ihrer Außenlegitimität und damit ihrer Ressourcenbasis zunehmend in den Sog von Politik und Wirtschaft" (Reinisch 2009, 13). Ähnlich Lisop $(2009,6)$ : „Betrachten wir die Berufs- und Wirtschaftspädagogik im Spiegel historischer Etappen, dann sehen wir deutlich, dass ihre Themen, ihre jeweilige institutionelle Stabilität, Stärke oder Schwäche und auch die wissenschaftliche Selbstreflexion bzw. Identität stark an die allgemeine Politikentwicklung angelehnt waren, speziell an deren ökonomische Basis“.

3 Vgl. neuerdings die „Antworten auf die Kritiker“ (Baumert/Tillmann 2017). 
Trotzdem ist die Berufs- und Wirtschaftspädagogik keine politisch reaktive Disziplin. Die Beziehung zwischen systematischem Wissen und politischem Handeln ist nicht einseitig, vielmehr handelt es sich „um ein Bedingungsverhältnis zwischen unterschiedlichen Konfigurationen politischer Zielformulierung und wissenschaftlicher Entwicklung (Weingart 1976, 167). So werden beispielsweise Forschungsförder- und Berufsbildungspolitik von Ausschüssen, Gremien, informellen Beratungen gesteuert, an denen die Berufs- und Wirtschaftspädagogik beteiligt ist. Deshalb ist weniger die Frage relevant, ob sie an Politik partizipiert, sondern vielmehr, in welcher Weise sie mit ihr verflochten ist, welchen Einfluss sie hat und wer festlegt, was als vernünftiges und richtiges Handeln gelten kann, inwieweit sie jenseits übergreifender Orientierungen von sich aus Probleme auf die politische Agenda setzt, Meinungen mitproduziert, in welchem Verhältnis wissenschaftliche und politische Probleme zueinander stehen, und wie die Übersetzungen zwischen beiden im konkreten Beratungsprozess ablaufen (vgl. Weingart 2003, 92). Für die Berufs- und Wirtschaftspädagogik ist die Verflochtenheit mit Politik, das Einsickern politischer Strömungen in die innere Wissenschaftsstruktur und ihre Einflussnahme auf politische Steuerungen und Entscheidungen schwer rekonstruierbar. Nicht unwesentlich ist in diesem Zusammenhang auch die Frage, wie und wodurch die Wissensproduktion im disziplinären Binnenverhältnis gelenkt wird, inwiefern und welche disziplinären Diskursregime durch Forschungs- und Berufsbildungspolitik zustande kommen, die theoretische und methodische Reflexionen oder Nicht-Reflexionen steuern, Probleme, Programme, Instrumente der Forschung als richtig und wichtig definieren, festlegen, was Plausibilität beanspruchen kann und was nicht, was oder wessen Positionen als wahr und legitim gelten und welche nicht, was der „Jargon der Eigentlichkeit" ist, welche Denkstile in die Hochschullehre hineingetragen werden und die inhaltliche Ausrichtung von Professuren bestimmen. Fraglich ist auch, ob und inwieweit disziplininterne Abschottungen von Forschungssträngen der Logik von forschungsförder- und berufsbildungspolitischen Abhängigkeiten folgen. Die Verstricktheit der Berufs- und Wirtschaftspädagogik mit Politik muss aber nicht zwangsläufig auf Kosten der Binnenlegitimität gehen, nämlich dann nicht, wenn die politische Involviertheit der disziplinären und wissenschaftlichen Kritik zugänglich wird (vgl. Reinisch 2009, 13).

\section{Selbstverständnis über Grundbegriffe}

Die Verständigung über disziplinäre Grundbegriffe, deren Gehalt und Widersprüche, bildet eine Grundlage für Selbstverständnisdiskurse. Zu den Grundkategorien der Berufs- und Wirtschaftspädagogik gehören zunächst die im 
Disziplinnamen enthaltenen Begriffe: Beruf, Wirtschaft, Pädagogik bzw. Bildung.

\subsection{Beruf}

Beruf ist eine Zentralkategorie der Berufs- und Wirtschaftspädagogik. Kritische Auseinandersetzungen mit dem Beruf tangieren seit der zweiten Hälfte des 20. Jahrhunderts auch die Selbstverständnisfrage. Da der Beruf seit der Industrialisierung in Frage steht (vgl. Abel 1963), ist ein berufs- und wirtschaftspädagogischer identitätsbildender Kern seit Disziplingründung fragil. Dass die Berufsbildungstheorie und die durch sie beeinflusste Berufs- und Wirtschaftspädagogik vor der heterogenen und widersprüchlichen Berufswirklichkeit lange Zeit die Augen verschlossen und sich dabei ,auf merkwürdige Mystifizierungen, auf Unterscheidungen von echtem und unechtem Beruf" (Stratmann 1975, 307) eingelassen haben, wurde ihr in den 1970er Jahren ,als eine der schwersten Hypotheken“ (ebd., 306) vorgeworfen. Noch zwanzig Jahre später schreibt Kutscha (1992, 538): „Die Berufs- und Wirtschaftspädagogik täte mithin gut daran, sich mit den Widersprüchen und Paradoxien auseinanderzusetzen, die aus der Gleichzeitigkeit disparater Entwicklungen bzw. Entwicklungszustände resultieren“. Grund für die Berufsproblematik ist die Diskrepanz zwischen einem abstrakten Beruf und den konkreten Inhalten von Arbeit. Berufe sind nicht logische Folgerungen von Arbeitsprozessen, sondern Ergebnisse sozialer Aushandlungen, in die mit Bildungsansprüchen interveniert werden könnte. Eine politikorientierte Perspektive haben in den 1980er Jahren die Vertreter der gestaltungsorientierten Berufsbildungsforschung (vgl. Rauner 1985) eingenommen, um im Sinne von Sozialverträglichkeit und Mitbestimmung Planungsprozesse von Berufen zu hinterfragen, die für Berufe charakteristischen Aufgaben zu identifizieren und $\mathrm{zu}$ modellieren. Anstatt diese Perspektive berufsfeldübergreifend $\mathrm{zu}$ diskutieren, verblieb sie im engen Kreis berufswissenschaftlicher Forschung und ihrer gewerblich-technischen Fachdidaktiken. Ansonsten dominierte in der Disziplin ein sachlogisch begründetes evolutionäres Berufsdenken, das sich in verschiedenen Diskurslinien mit unterschiedlichen Szenarien niederschlug. So wurden eine Erosion, eine Dysfunktionalität und eine bedingte Stabilität des Berufs prognostiziert, auch verbunden mit Vorschlägen sich auf andere Kategorien wie Arbeit und Erwerb zu konzentrieren oder über institutionelle und individuelle Flexibilisierungsformen nachzudenken. Neben unkritischen Pauschalrezeptionen von Megatrends sind für die meisten Auseinandersetzungen mit Beruf auch ,pauschale Aussagen über die Berufe, die Beruflichkeit, die Entberuflichung, das Berufskonzept" (Kell 2016, 1) kennzeichnend, so dass kaum Anknüpfungspunkte für erziehungswissenschaftliche, curriculare und didaktische Überlegungen offengelegt werden können. 
Selbstbeschränkend in der Diskussion um Beruf war auch lange Zeit die Fixierung auf das Duale System der Berufsausbildung. Berufsrelevante Entwicklungen jenseits dieser Sphäre, also im Weiterbildungssektor, im Übergangsbereich oder im Hochschulsystem, sind daher schnell als Bedrohung des als eigentlich wahrgenommenen Berufs interpretiert worden. Möglicherweise hätten durch eine weniger standesfixierte Berufsbezogenheit viel früher „Beruf und Wissenschaft als organisierende Prinzipien“ (Reetz 1976) mit Blick auf Akademisierung der Berufsbildung ausgelegt werden können. Vielleicht wäre dann auch die Aufgeschlossenheit für Ideen, wie die der ,erweiterten Beruflichkeit" (Kutscha 2015) mit der Perspektive integrierter Berufsund Bildungskonzepts, größer.

\section{2 Ökonomie}

Die Berufs- und Wirtschaftspädagogik hat es in mehrfacher Hinsicht mit dem Ökonomischen zu tun, als äußere Bedingung, wenn es um die Frage effizienter berufsbildungspolitischer und -institutioneller Steuerung geht, und als Gegenstand, wenn curriculare und fachdidaktische Frage des Wirtschaftsunterrichts verhandelt werden (vgl. Bellmann 2001, 228ff). Das, was beide Ebenen miteinander verbindet, sind grundsätzliche Auffassungen von Ökonomie, über die sich Berufs- und Wirtschaftspädagogik zu verständigen hat, wenn sie Lehrerinnen und Lehrer dazu befähigen will, aus einer „ethisch legitimierten und selbstreflexiv kontrollierten Perspektive Urteile zu bilden“" (Sektion Berufs- und Wirtschaftspädagogik 2014, 5). In der Berufs- und Wirtschaftspädagogik hat sich im Verlauf ihrer Geschichte das Verständnis von Wirtschaft mit den jeweiligen Denkstilen und Theoriebezügen verändert. So verstand Feld $(1928,15)$ Wirtschaft im Kontext seiner Kultur- und Wertphilosophie weniger als „Sphäre des Egoismus“ und „Welt der Ausbeutung“, sondern als „Kultursphäre“. Für Ausbeutung, Missstände in der Bildung und für die Lehrlingszustände dürfe ,nicht die Wirtschaft an sich“ (ebd., 16) verantwortlich gemacht werden, ,sondern die Menschen, die sich ihrer Verantwortung der Gemeinschaft gegenüber entziehen“ (ebd.). Für ihn stand fest, „daß die Wirtschaft ihre eigenen Gesetze hat und betriebswirtschaftliches Denken und Forschen an sich mit der Ethik nichts zu tun hat" (ebd., 18). Dennoch müsse „Wirtschaftlichkeit als Norm unseres werktätigen Lebens und Handels" (ebd., 21) verstanden werden, damit der Mensch dem Wirtschaftsprozess nicht feindlich gegenübersteht, sondern ihn begreift und als „Teilfunktion“ in Gesellschaft und Betrieb hinnimmt. Das Ziel der „wirtschaftlichen Bildung" liege im Verständlich machen der gegebenen wirtschaftlichen Vorgänge (vgl. ebd., 27). Schlieper $(1963,110)$ grenzte sich von der Auffassung ab, dass ,das ,wirtschaftliche“ Handeln eigene unabhängige Normen und Gesetze beanspruche“ und stellte heraus, dass die „Wirtschafts- 
form“ (ebd.) der Wirtschaftsauffassung, „die sie beherrscht“ (ebd.), entspricht. Verschiedene Wirtschaftsauffassungen wurden von ihm nicht weiter reflektiert, vielmehr folgert er, dass ,,je nachdem, zu welcher erziehungswissenschaftlichen Richtung sich die einzelnen Pädagogen bekennen, [...] sie die Bedeutung des wirtschaftlichen Lebens- und Kulturbereiches für die Erziehung anders einschätzen [werden]“ (ebd., 113). Abraham $(1967,259)$ wies auf die Tendenz der Funktionalisierung des Menschen durch die Wirtschaft hin und insistierte darauf, dass es nicht nur um ,ökonomische Brauchbarkeit“ gehen dürfe, sondern, ,die Kernfrage lautet vielmehr, wie der [moderne Mensch] unter den Existenzbedingungen, die [...] von der Wirtschaft aufgezwungen werden, sein Leben so gestalten kann, daß es nicht nur ökonomisch erfolgreich ist, sondern ihn auch geistig befriedigt". Mit seinen Überlegungen zu „Wirtschaft im Bildungsraum“ wird auch bei ihm ein Verständnis von Wirtschaft als übergreifende Gegebenheit deutlich: „Es ist für die Wirtschaft lebenswichtig, daß sich das Erziehungswesen in Ordnung befindet [und] daß der moderne Mensch innerlich so stark [ist], daß er die innere Freiheit behält, ohne die das gesamte Dasein wertlos ist“" (ebd., 271).

Eine politische Perspektive auf Ökonomie findet sich hingegen bereits bei Fischer (1932/1963, 35): „Ökonomische Kategorien beherrschen heute alles und alle. Man muß es aus dieser uneingeschränkten Vorherrschaft des Ökonomismus in Denkhaltung und Lebenseistellung verstehen, wenn auch der Mensch und seine Bildung dem Geltungs- und Machtbereich der Wirtschaft unterworfen werden. Der Mensch verliert dabei seine Würde, wird Ware". Er thematisierte die durch wirtschaftliche Macht hervorgerufene Ungleichheit, den „Gegensatz zwischen Luxus auf der einen und halszuschnürender Not auf der anderen Seite, die Extreme der Massenverelendung und des sinnlosen Luxus, die Härte eines Rechtes, das solche Extreme gewiß ursprünglich nicht gewollt hat, aber tatsächlich schützt, die Entfremdung der Menschen“ (S. 53). Aus seinen Überlegungen zog er die Konsequenz, die Bildungskräfte des Wirtschaftslebens im „Geist des sozialen Humanismus“ (S. 58) und gerechter Arbeits- und Wirtschaftsordnung (S. 56) zu identifizieren und deren Entfaltung zur Aufgabe aller Schulformen zu machen. Ähnlich kritische Positionen finden sich später bei Linke (1957/1967). Die „Prosperität der Wirtschaft“ sei nicht Maß aller Dinge. Diese dürfe ,immer nur den Charakter eines Zweckmittels“ (ebd.) besitzen. Das Augenmerk „wirtschaftspädagogischer Forschung“ habe sich ,,auf die Gestaltbarkeit der Wirtschaft" (S. 129) zu richten. Er kritisierte auch den ,Zirkel der engen Verflechtung zwischen wirtschaftlicher Erfolgsrechnung und berufspädagogischer Bildungsplanung“" (Linke 1965, 326). Auch Dörschel $(1960,103)$ wollte das „ökonomische Bildungsideal“ aus „,der Idee und dem Phänomen der Menschlichkeit“ und der „Humanisierung der Arbeit“ und als „Antithese zu bestehenden politisch-ideologischen Glaubens- und Denkmustern“ begreifen. Mit gesellschafts- und bildungskritischem Anspruch verfasste Kutscha 
(1976) seinen politisch-ökonomischen Strukturgitteransatz, um durch politisch-ökonomische Bildung im Medium des Berufs eine Grundlage zu bieten, um Bedingungen und Folgen wirtschaftlicher Entscheidungen und Handlungen im Kontext politischer und ethischer Problemstellungen differenziert und kritisch beurteilen zu können. In der Berufs- und Wirtschaftspädagogik gibt es also schon seit längerem bildungskritische Überlegungen, die das „Ökonomische“ nicht mehr vorrangig als ,(materiales) medium quod“ (Bellmann 2001, 236), sondern als ,(formales) medium quo der Bildung“ (ebd.) verstehen. Die Frage ist, welchen disziplinären Stellenwert diese Überlegungen noch oder wieder haben. Oder führen aktuelle Diskursregime zurück zur ökonomischen Sachlogik? Die Überwindung des schon von Fischer (1932/1963) formulierten „Ökonomismus“ gehört heute angesichts der aktuellen Debatten um Ökonomisierung als soziale Regulierungsform zu wichtigen Themen des berufs- und wirtschaftspädagogisches Diskurses, in dessen Zusammenhang auch anders über „ökonomische Kompetenz" diskutiert werden und eine kritische Reflexion von Individualmoral in der Marktwirtschaft und Wirtschaftsethik erfolgen müsste.

\subsection{Bildung}

Im Basiscurriculum der Sektion für Berufs- und Wirtschaftspädagogik (2014) taucht an mehreren Stellen das Wort „bildungstheoretisch“ auf. Die Frage ist, was in der Berufs- und Wirtschaftspädagogik damit gemeint ist. Die klassische berufsbildungstheoretische Grundidee, dass Bildung über den Weg von Arbeit und Beruf erfolgen kann, wurde in der neuhumanistischen Bildungstheorie angezweifelt, da die Frage nach der bildenden Kraft des Berufs immer auch eine Frage nach der Beschränkung des Menschen und der Enge von Berufsarbeit, an die er gebunden ist, war (vgl. Stratmann 1979/1999, 524). Die Skepsis gegenüber einer Bildung, die das NS-Regime nicht verhindern konnte und für die nun alle Voraussetzungen zerstört waren (vgl. Litt 1947, $12 \mathrm{f}$.), und gleichzeitig der Anspruch, die Berufs- und Arbeitswirklichkeit in der Berufsbildungsdiskussion nicht mehr zu ignorieren, führten nach 1945 dazu, sich entweder ganz vom Bildungsbegriff zu verabschieden oder ihn unter gesellschaftskritischem Aspekt zu begreifen. So war Abel (1963) der Ansicht, dass Bildung durch den Beruf „kein Zauberwert zur Rettung der Menschen“ (S. 196) mehr sei, während für Wilhelm $(1966,97)$ „das Schema ,Bildung durch den Beruf" nicht mehr glaubwürdig" war. Blankertz $(1967 / 75,296)$ räumte ein, dass über Bildung nur noch unter kritischem Vorbehalt gedacht werden könnte und die Berufsbildungstheorie ohnehin „durch den Wandel der Ausbildungsbedingungen beeinträchtigt wird“. Die Rettung der Bildung bei Blankertz erfolgte dann mit dem Hinweis, dass die Begrenzungen von Bildung sie wiederum dazu ermächtigen, ,unter neuen Bedin- 
gungen als organisierende Kraft die Ausbildungskonzeption pädagogisch zu strukturieren" (ebd.), ein Gedanke, der für den bildungstheoretischen Strukturgitteransatz maßgeblich war. Von dem bildungstheoretischen und -kritischen Diskurs dieser Zeit grenzten sich jene Positionen ab, die sich mehr als kognitiv-pragmatisch oder empirisch-analytisch verstehen wollten und auch eher den Erziehungsbegriff bevorzugten. Wie in Teilen der Erziehungswissenschaft wurde auch in der Berufs- und Wirtschaftspädagogik der Erziehungsbegriff als „Oberbegriff mit systembildender Bedeutung“ (Dörschel 1960, 35) favorisiert, dem ,andere erziehungswissenschaftliche Begriffe wie Bildung, Führung, Zucht, Pflege, Berufserziehung, -bildung und -ausbildung ausdrücklich untergeordnet" wurden. In den folgenden Jahrzehnten ist der Bildungsbegriff in der Berufs- und Wirtschaftschaftspädagogik immer mehr in den Hintergrund getreten. Damit sind auch berufsbildungstheoretische Diskurse „aus der Mode gekommen“ (Kutscha 2009, 14). Dies mag auch der Grund dafür sein, dass in berufs- und wirtschaftspädagogischen Einführungsschriften zur Didaktik, „die Rezeption differenzierter [Bildungs] theorien“ abbricht, „,bevor sie konkret (oder ,praktisch') werden könnte“ (Greb 2009, 21).

Eine wesentliche Bezugsgröße bildungstheoretischer Wiederbelebung könnte das Subjektverständnis sein, zumal universelle Form und Zielperspektive verschiedener berufs- und wirtschaftspädagogischer Bemühungen das souveräne Subjekt ist, das autonom Entscheidungen trifft und sich selbst entfaltet. Trotz des bildungstheoretischen Rückzugs ist der Subjektbegriff in der Berufs- und Wirtschaftspädagogik also nach wie vor beliebt, jedoch weniger auf einer theoretisch-analytischen, sondern vielmehr auf einer normativen Ebene. Thoma (2011) arbeitete in „Entwürfe des wirtschaftspädagogischen Subjekts" heraus, dass unklar bleibt, wie das Subjekt konzeptualisiert ist, und wie es durch berufs- und wirtschaftspädagogisch wohl gemeinte subjektorientierte Programme erneut in Machtzentren eingespannt wird. Die im Subjektbegriff liegenden Spannungen und Widersprüche werden in Didaktik- und Kompetenzkonzepten kaum thematisiert. Dabei liegt das, „was ein Subjekt ist, [...] nicht ein für alle Mal fest, sondern lässt sich nur erschließen über die historischen Semantiken und Wissenskomplexe, die Selbst- und Sozialtechnologien, die zu seiner theoretischen Bestimmung und praktischen Formung aufgerufen wurden und werden“ (Bröckling 2007, 23). Somit erweist sich auch die Gegenüberstellung von objektiven Anforderungen auf der einen Seite und Subjekt auf der anderen Seite als zu einfach. In den ,individuellen Bildungsbedürfnissen [kommen] auf so vielfältige und nachhaltige Weise gesellschaftliche, ökonomische und am Ende eben auch betriebliche Anforderungen zur Geltung [...], ja sie sind geradezu inkorporiert, so daß die pädagogisch-idealistische Kontrastierung zwischen [...] ökonomischen Anforderungen einerseits und individuellen Bildungsbedürfnissen andererseits unrealistisch, um nicht zu sagen, als ideologisch interpretiert werden kann“" (Heid 1998, 45). Deshalb geht es in der bildungstheoreti- 
schen Reflexion von Subjekt auch um die Frage, wie Lernende mit wirtschaftlichen, politischen und gesellschaftlichen Denkformen verwoben sind, wie sich diese als Orientierungen in Lebenswelten, „mentalen Infrastrukturen“ (Welzer 2011) und subjektiven Bewertungs- und Verteilungsmaßstäben niederschlagen. Eine Wiederbelebung und Modernisierung des bildungstheoretischen Diskurses könnte dies thematisieren, ebenso die Frage, inwiefern die Disziplin selber von Denkformen gesteuert ist, die sich als Machtformen in ihre subjektorientierten Konzepte und Messforschung einschleichen.

\section{Fazit}

Anliegen des Beitrags ist die Wiederbelebung des Selbstverständnisdiskurses in der Berufs- und Wirtschaftspädagogik. Mögliche Anknüpfungspunkte hierfür können der historische Verlauf der Selbstverständnisfrage, die darin kontinuierlich thematisierten neuralgischen Punkte sowie die Spannungen und Widersprüche disziplinärer Grundkategorien sein.

\section{Literatur}

Abel, H. (1963). Das Berufsproblem im gewerblichen Ausbildungs- und Schulwesen. Braunschweig: Westermann.

Abraham, K. (1953). Die Erziehung des Menschen in der Wirtschaft. In Die Deutsche Berufs- und Fachschule, 49, 321-329.

Abraham, K. (1957). Die Bedeutung der begrifflichen Klarheit für die wirtschaftspädagogische ForschungIn Die Deutsche Berufs- und Fachschule, 53, 169-175.

Albert, H. (1991). Traktat über kritische Vernunft, 5. Auflage, Stuttgart: UTB.

Bank, V. (2009): Berufs- und Wirtschaftspädagogik: Epitaph einer Disziplinlosen. bwp@ Berufs- und Wirtschaftspädagogik - online, 16, 1-22. Online: http://www. bwpat.de/ausgabe 16/bank_bwpat16.pdf (27-12-2016).

Baumert, J. \& Tillmann, J. (2017). Empirische Bildungsforschung. Zeitschrift für Erziehungswissenschaft- Sonderheft 31. Wiesbaden: Springer VS.

Baumgardt, J. (1976). Entwicklung und Stand der Wirtschafts- und Berufspädagogik. In Schanz, H. (Hrsg), Entwicklung und Stand der Berufs- und Wirtschaftspädagogik (S. 7-38). Stuttgart: Holland \& Josenhans.

Beck, K. (2003). Erkenntnis und Erfahrung im Verhältnis zu steuerung und Gestaltung. Zeitschrift für Berufs- und Wirtschaftspädagogik, 99, 232-250.

Bellmann, J. (2001). Knappheit als Bildungsproblem. Weinheim: Deutscher Studienverlag.

Blankertz, H. (1967). Berufsbildungstheorie und berufliche Ausbildungskonzeption. In K. Stratmann \& W. Bartel (Hsrg.). a.a.O. Wiederabdruck (S. 285-299). 
Bröckling, U. (2007). Das unternehmerische Selbst. Soziologie einer Subjektivierungsform. Frankfurt a. M.: Suhrkamp.

Dammer, K.-H. (2015). Vermessene Bildungsforschung. Wissenschaftsgeschichtliche Hintergründe zu einem neoliberalen Herrschaftsinstrument. Baltmannsweiler: Schneider.

Deutsche Forschungsgemeinschaft (Hrsg.) (1990). Berufsbildungsforschung an den Hochschulen der Bundesrepublik Deutschland. Denkschrift. Weinheim: Beltz.

Dörschel, A. (1960). Einführung in die Wirtschaftspädagogik. Berlin und Frankfurt a.M.: Franz Vahlen GmbH.

Feld, F. (1928). Grundfragen der Berufsschul- und Wirtschaftspädagogik. Versuch einer Systematik der berufspädagogischen Theorie. Langensalza: Julius Beltz.

Fischer, A. (1932). Wirtschaft und Schule. In H. Röhrs (Hrsg) (1963). Die Bildungsfrage in der modernen Arbeitswelt. Wiederabdruck (S. 35-60). Frankfurt am Main: Akademische Verlagsgesellschaft.

Geck, L.H.A. (1932). Zur Grundlegung der Wirtschaftspädagogik. Zeitschrift für Handelsschulpädagogik, 4, 145-158 und 220-231.

Greb, U. (2009): Der Bildungsbegriff in einführenden Schriften zur Didaktik der Berufs und Wirtschaftspädagogik.bwp@Berufs- und Wirtschaftspädagogik online, 16, 1-23. Online: http://www.bwpat.de/ausgabe16/greb_bwpat16.pdf (2712-2016).

Groothoff, H.H. (1961). Allgemeine und Wirtschaftspädagogik. Die Deutsche Berufsund Fachschule, 57 (10), 772-781.

Heid, H. (1977). Können „die Anforderungen der Arbeitswelt“ Ableitungsvoraussetzungen für Maßgaben der Berufserziehung sein? Die Deutsche Berufs- und Fachschule, 73 (11), 833-839.

Heid, H. (1998): Berufliche Bildung im Spannungsfeld zwischen betrieblichen Anforderungen und individuellen Ansprüchen. In Berufliches Lernen im Wandel - Konsequenzen für die Lernorte? Dokumentation des 3. Forums Berufsbildungsforschung (33-54). Nürnberg: MitAB.

Kautz, H. (1929). Industrie formt Menschen. Einsiedeln: Verlagsanstalt Benzinger \& Co.

Kell, A. (2014). Grenzgänge, Traditionen und Zukünfte in der Deutschen Gesellschaft für Erziehungswissenschaft. Erziehungswissenschaft. Mitteilungen der DGfE, 25 (49), 49-64.

Kell, A. (2015). Arbeit und Beruf aus Sicht ökologischer Berufsbildungswissenschaft. bwp@Berufs- und Wirtschaftspädagogik - online, Ausgabe 29, 1-25. Online: http://www.bwpat.de/ausgabe29/kell_beitrag1_bwpat29.pdf(27-12-2016).

Kiehn, L. (1962). Gedanken zur wissenschaftstheoretischen Grundlegung der Wirtschafts- und Berufspädagogik. In H. Röhrs (Hrsg.) (1967). Die Wirtschaftspädagogik - eine erziehungswissenschaftliche Disziplin? Wiederabdruck (S. 36-43). Frankfurt a.M.: Akademische Verlagsgesellschaft.

Kipp, M. \& Miller-Kipp, G. (1994). Kontinuierliche Karrieren. Diskontinuierliches Denken? Zeitschrift für Pädagogik, 40 (5), 727-744.

Krasensky, H. (1935). Grundzüge der Wirtschaftspädagogik. Wien: Heymann Verlag.

Kutscha, G. (1992). ,Entberuflichung' und ,Neue Beruflichkeit'. Zeitschrift für Berufs- und Wirtschaftspädagogik, 88, 535-548.

Kutscha, G. (2009). Bildung im Medium des Berufs? In I. Lisop \& A. Schlüter (Hrsg.), a.a.O. (S. 13-36). 
Kutscha, G. (2015). Erweiterte moderne Beruflichkeit. bwp@ Berufs- und Wirtschaftspädagogik - online, 29, 1-22. Online: http://www.bwpat.de/ausgabe29/ kutscha_bwpat29.pdf (27-12-2016).

Kutscha, G. (1976). Das politisch-ökonomische Curriculum. Kronberg/Ts: Fischer Taschenbuch.

Lange, H. (1971). Technik und Praxis. Bemerkungen zum wissenschaftstheoretischen und politischen Standort einer zeitgemäßen Berufs- und Wirtschaftspädagogik. In K. Stratmann \& W. Bartel (Hrsg.). a.a.O. Wiederabdruck (S. 308-337).

Lempert, W. (1972). Vorüberlegungen zum theoretischen Rahmen wissenschaftlicher Analysen beruflicher Bildungsprozesse. In K. Stratmann \& W. Bartel (Hrsg.) a.a.O. (338-349).

Lempert, W. (2009). Die Fliege im Fliegenglas, der Globus von Deutschland und die Berufsbildung ohne Beruf.bwp@Berufs-und Wirtschaftspädagogik-online, 16, 1-45. Online: http://www.bwpat.de/ausgabe16/lempert_bwpat16.pdf (17-122016).

Linke, W. (1957). Gegenstand und Standort der wirtschaftspädagogischen Forschung. Die Deutsche Berufs- und Fachschule, 53 (10), 741-753.

Linke, W. (1961). Kritische Beiträge zur Wirtschaftspäsagogik. Die Deutsche Berufsund Fachschule, 57 (10), 737-738.

Linke, W. (1965). Pädagogischer Erfahrungsaustausch in der praktischen und theoretischen Berufserziehung. Die Deutsche Berufs- und Fachschule, 69 (5),326.

Lipsmeier, A. (1972). Vom Beruf der Berufspädagogen. Zur Wissenschaftstheorie der Berufspädagogik. Die Deutsche Berufs- und Fachschule, 68 (1), 21-49.

Lisop, A. \& Schlüter, A. (Hrsg.) (2009). Bildung im Medium des Berufs? Diskurslinien der Berufs- und Wirtschaftspädagogik. Frankfurt a. M.: G.A.F.B.-Verlag.

Lisop, I. (2009): Identität und Krisenanfälligkeit der Berufs- und Wirtschaftspädagogik.bwp@Berufs-und Wirtschaftspädagogik - online, 16, 1-18. Online: http:// www.bwpat.de/ausgabe16/lisop_bwpat16.pdf (27-12-2016).

Neumann, G. (1969). Die Indoktrination des Nationalsozialismus in der Berufserziehung. Hamburg: Universität Hamburg.

Pleiß, U. (1971). Friedrich Feld und die Begründung der Wirtschaftspädagogik. In Pleiß, U. (Hrsg) (1982), Wirtschaftspädagogik, Bildungsforschung, Arbeitslehre. Wiederabdruck (S. 195-208). Heidelberg: esprint Verlag.

Pleiß, U. (1973). Wirtschaftslehrerbildung und Wirtschaftspädagogik. Göttingen: Schwartz.

Rauner, F. (1985). Technik und Bildung. In Diskurs. Bremer Beiträge zu Wissenschaft und Gesellschaft (S. 110-131), Band 10. Universität Bremen.

Reetz, L. (1976). Beruf und Wissenschaft als organisierende Prinzipien des wirtschaftslehre-Curriculums. Die Deutsche Berufs- und Fachschule, 72 (11), 803818.

Reinisch, H. (2009): Über Nutzen und Schaden des Philosophierens über das Selbstverständnis der Berufs- und Wirtschaftspädagogik. bwp@, Berufs- und Wirtschaftspädagogik - online, 16, 1-17. Online: http://www.bwpat.de/ausgabe16/ reinisch_bwpat16.pdf (27-12-2016).

Ritzel, W. (1961). Aktuelle Fragen der Wirtschaftspädagogik und des wirtschaftspädagogischen Studiums. Die Deutsche Berufs- und Fachschule, 57 (10), 738-753.

Schlieper, F. (1949). Die Lehre von der Wirtschaftserziehung. Die Deutsche Berufsund Fachschule, 45 (1), 42. 
Schlieper, F. (1952). Wesen und Inhalt einer Lehre von der Wirtschaftserziehung. Die Deutsche Berufs- und Fachschule, 48 (5), 423.

Schlieper, F. (1954). Grundbegriffe der Wirtschaftspädagogik. In H. Röhrs (Hrsg.) (1967). Die Wirtschaftspädagogik - eine erziehungswissenschaftliche Disziplin? (S. 54-74). Wiederabdruck. Frankfurt a.M.: Akademische Verlagsgesellschaft.

Schlieper, F. (1963). Allgemeine Berufspädagogik. Freiburg im Breisgau: Lambertus.

Schmiel, M.; Sommer, K.-H. (2001). Berufs- und Wirtschaftspädagogik als wissenschaftliche Disziplin. In H. Schanz (Hrsg.). Berufs- und wirtschaftspädagogische Grundprobleme (S. 8-21). Baltmannsweiler: Schneider.

Schürholz, F. (1928). Grundlagen einer Wirtschaftspädagogik. Zum Kampf um Wirtschaftsführung und Sozialordnung. Erfurt: K. Stenger.

Schwarzlose, A. (1961). Empirische Forschung in der Wirtschaftspädagogik. Die Deutsche Berufs- und Fachschule, 57 (10), 753-768.

Seubert, R. (1977). Berufserziehung und Nationalsozialismus. Weinheim: Beltz.

Stratmann, J. (1979). Berufs- und Wirtschaftspädagogik. In G. Pätzold \& Wahle, M. (Hrsg.) (1999), Karlwilhelm Stratmann. Berufserziehung und sozialer Wandel. Wiederabdruck (S. 509-581). Frankfurt a.M.: Gesellschaft zur Förderung arbeitsorientierter Forschung und Bildung Verlag.

Stratmann, K. \& Bartel, W. (Hrsg.) (1975). Berufspädagogik. Ansätze zu ihrer Grundlegung und Differenzierung. Köln: Kiepenheuer \& Witsch.

Stratmann, K. (1972). Zum Thema dieses Heftes: Das Theorie-Praxis-Verhältnis in der Berufs- und Wirtschaftspädagogik. Die Deutsche Berufs- und Fachschule, 68 (8), 575-577.

Stratmann, K. (1975). Historische Pädagogik als Mittel der Entmythologisierung und Entideologisierung. In J.L. Blaß u. a. (Hrsg.), Bildungstradition und moderne Gesellschaft, (S. 304-322). Hannover: Schrödel.

Tenorth, H.E. (2004). Erziehungswissenschaft. In D. Benner \& J. Oelkers (Hrsg.). Historisches Wörterbuch der Pädagogik (S. 341-382). Weinheim: Beltz.

Thoma, M. (2011). Entwürfe eines wirtschaftspädagogischen Subjekts. Wiesbaden: VS Verlag.

Weingart, P. (1976): Wissensproduktion und soziale Struktur. Frankfurt am Main: Suhrkamp.

Weingart, P. (2003). Wissenschaftssoziologie. Bielefeld: transcript.

Welzer, H. (2011). Mentale Infrastrukturen. Heinrich Böll Stiftung. Online: https:// www.boell.de/sites/default/files/Endf_Mentale_Infrastrukturen.pdf (20-12-2016).

Wilhelm, T. (1966). Das Arbeitsethos der Gegenwart im Lichte der deutschen Bildungsüberlieferung. In K. Stratmann \& W. Bartel (Hrsg.) (1975), a.a.O., Wiederabdruck (S. 95-111).

Zabeck, J. (1968). Zur Grundlegung und Konzeption einer Didaktik der kaufmännischen Berufserziehung. In Jahrbuch für Wirtschafts- und Sozialpädagogik (S. 87141). Heidelberg: Quelle \& Meyer.

Zabeck, J. (1972). Das Theorie-Praxis-Problem in der Wirtschaftspädagogik. Die Deutsche Berufs- und Fachschule, 68 (8), 577-599. 